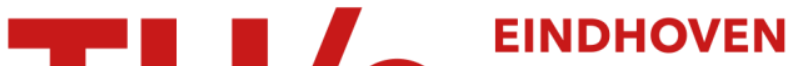 UNIVERSITY OF TECHNOLOGY
}

\section{Approximating non-spherical singularities as spherical singularities for objects at an interface between two layers}

\section{Citation for published version (APA):}

Sakai, S., Tijhuis, A. G., \& Van Beurden, M. C. (2016). Approximating non-spherical singularities as spherical singularities for objects at an interface between two layers. In Proceedings of the 2016 18th International Conference on Electromagnetics in Advanced Applications, ICEAA 2016 (pp. 512-515). [7731442] Institute of Electrical and Electronics Engineers. https://doi.org/10.1109/ICEAA.2016.7731442,

https://doi.org/10.1109/ICEAA.2016.7731442

DOI:

10.1109/ICEAA.2016.7731442

10.1109/ICEAA.2016.7731442

Document status and date:

Published: 01/09/2016

\section{Document Version:}

Publisher's PDF, also known as Version of Record (includes final page, issue and volume numbers)

\section{Please check the document version of this publication:}

- A submitted manuscript is the version of the article upon submission and before peer-review. There can be important differences between the submitted version and the official published version of record. People interested in the research are advised to contact the author for the final version of the publication, or visit the $\mathrm{DOI}$ to the publisher's website.

- The final author version and the galley proof are versions of the publication after peer review.

- The final published version features the final layout of the paper including the volume, issue and page numbers.

Link to publication

\section{General rights}

Copyright and moral rights for the publications made accessible in the public portal are retained by the authors and/or other copyright owners and it is a condition of accessing publications that users recognise and abide by the legal requirements associated with these rights.

- Users may download and print one copy of any publication from the public portal for the purpose of private study or research.

- You may not further distribute the material or use it for any profit-making activity or commercial gain

- You may freely distribute the URL identifying the publication in the public portal.

If the publication is distributed under the terms of Article 25fa of the Dutch Copyright Act, indicated by the "Taverne" license above, please follow below link for the End User Agreement:

www.tue.nl/taverne

Take down policy

If you believe that this document breaches copyright please contact us at:

openaccess@tue.nl

providing details and we will investigate your claim. 


\title{
Approximating non-spherical singularities as spherical singularities for objects at an interface between two layers
}

\author{
Satoru Sakai* Anton G. Tijhuis ${ }^{\dagger} \quad$ Martijn C. van Beurden ${ }^{\ddagger}$
}

\begin{abstract}
A mathematical approach to evaluate the singularity present in the dyadic Green function for objects close to an interface is presented in this paper. This approach results in a spherically symmetric expression for the off-diagonal singular terms of the dyadic Green function. The main advantage is that the singularity no longer depends on the orientation of the RWG functions, and therefore only a uniform singularity needs to be extracted and evaluated. As a result, the implementation of this approach is more flexible, and also reduces the required coding effort.
\end{abstract}

\section{Introduction}

Singularities are present in the Green function of the electric field integral equation (EFIE) for a three-dimensional (perfectly conducting) scattering object. In order to obtain accurate solutions to the EFIE, care must be taken when evaluating integrals that involve these singularities. This is especially true when the scattering object is close to - or on top of - an interface between two media. For this case the matrix elements of the dyadic Green function become singular when the scatterer and the observation point are close to one another.

The EFIE for the scattering object is obtained by meshing its surface into triangles, where the Rao Wilton Glisson ([1], hereafter RWG) basis and test functions are employed to compute the unknown surface current density.

For layered media, [2] - and later on by [3] using a different representation - decompose the EFIE into scalar and vector potential (Green function) contributions, where the vector potential contains off-diagonal terms. The standard method for evaluating the contributions is to separate the function into two components: one regular component, which can be evaluated numerically, and one singular component, which can be evaluated analytically. The singularity for the scalar potential and the diagonal components of the vector potential

\footnotetext{
*Department of Electrical Engineering, Eindhoven University of Technology, P.O. Box 513, 5600 MB, Eindhoven, The Netherlands, email: s.sakai@tue.nl

${ }^{\dagger}$ Department of Electrical Engineering, Eindhoven University of Technology, The Netherlands

$\ddagger$ Department of Electrical Engineering, Eindhoven University of Technology, The Netherlands
}

can be extracted using image theory, which results in spherically symmetric singular contributions at the image position. However, for the off-diagonal components of the vector potential this approach leads to singular contributions of logarithmic type that lack spherical symmetry. As a result, in [4] the singular component of the kernel was written separately in the transverse and longitudinal coordinates, while others [5] have addressed the singular behaviour by suitably modifying the vector and scalar potential contributions.

In this paper we will describe how this singularity is derived and extracted in the literature. Then, a derivation which results in a spherical singularity is presented. Finally, a discussion of how to use this form to evaluate the self patch integral - resulting in a closed form integral expression - is given.

\section{EFIE evaluation}

The scattered electric field is obtained by integrating the scalar and vector potential Green function with the surface current density $\mathbf{J}$,

$\overline{\bar{L}}^{E J}=\nabla \phi+\mathbf{A}=\nabla \int G^{\phi} \nabla \cdot \mathbf{J} \mathrm{d} A^{\prime}+\int \overline{\bar{G}}^{A} \cdot \mathbf{J} \mathrm{d} A^{\prime}$.

The standard method for obtaining the surface current density $\mathbf{J}$ on the surface of the source (scattering object) is by meshing the surface with triangular patches described by [1]. This method produces test (hereafter $\overrightarrow{\mathrm{T}}(\mathbf{r})$ ) and basis (hereafter $\overrightarrow{\mathrm{B}}\left(\mathbf{r}^{\prime}\right)$ ) functions which are defined on a triangular mesh. Each function contains two triangles, represented by superscripted + and - , which share a common edge.

For the off-diagonal terms in the $z$-column of the dyadic Green function (for the vector potential), the integral expressed in [3] (Equation 58) is of the form

$$
\int_{T^{+} \cup T^{-}} \overrightarrow{\mathrm{T}}(\mathbf{r}) \cdot \nabla \int_{B^{+} \cup B^{-}} C^{\phi}\left(\mathbf{r}, \mathbf{r}^{\prime}\right)\left[\overrightarrow{\mathrm{B}}\left(\mathbf{r}^{\prime}\right) \cdot \hat{\mathbf{u}}_{z}\right] \mathrm{d} A_{B} \mathrm{~d} A_{T},
$$

where $\mathrm{d} A_{B}$ and $\mathrm{d} A_{T}$ are area elements of the basis and test function co-ordinate, respectively. Using Gauss's theorem, the quadrature expression for the 
off-diagonal components is

$$
\begin{aligned}
& -\iint_{T^{+} \cup T^{-}} \nabla \cdot \overrightarrow{\mathrm{T}}(r) \iint_{B^{+} \cup B^{-}} C^{\phi}\left(r, r^{\prime}\right)\left[\overrightarrow{\mathrm{B}}\left(r^{\prime}\right) \cdot \hat{\mathbf{u}}_{z}\right] \mathrm{d} A_{B} \mathrm{~d} A_{T} \\
& =\iint_{B^{+} \cup B^{-}}^{\overrightarrow{\mathrm{B}}}\left(r^{\prime}\right) \cdot \hat{\mathbf{u}}_{z}\left\{\iint_{T^{+} \cup T^{-}} C^{\phi}\left(r, r^{\prime}\right)[\nabla \cdot \overrightarrow{\mathrm{T}}(r)] \mathrm{d} A_{T}\right\} \mathrm{d} A_{B} .
\end{aligned}
$$

Since $\nabla \cdot \overrightarrow{\mathrm{T}}(r)$ is a constant on the test function, it is possible to analytically evaluate this component, and $\overrightarrow{\mathrm{B}}\left(r^{\prime}\right) \cdot \hat{\mathbf{u}}_{z}$ can be evaluated through quadrature. A similar quadrature expression can be obtained for the $z$-row of the dyadic Green function through reciprocity.

\section{Non-spherical singularity at an interface}

In free space, the vector and scalar potentials contain a singularity of the form $\frac{1}{R}$, where $R=$ $\sqrt{\left(x-x^{\prime}\right)^{2}+\left(y-y^{\prime}\right)^{2}+\left(z-z^{\prime}\right)^{2}}$, the unprimed co-ordinates denote the observation point and the primed co-ordinates is the source point. For this particular case, the vector potential, when written in matrix (or dyadic) form, contains only diagonal elements.

The presence of an interface (or layer) introduces off-diagonal terms in the vector potential of the dyadic Green function. This arises from the "correction term" in (Equation 47, [2]) where the expressions in the spectral domain contain $\frac{j k_{x}}{k_{\rho}^{2}}$ or $\frac{j k_{y}}{k_{\rho}^{2}}$ terms, where $k_{\rho}^{2}=k_{x}^{2}+k_{y}^{2}$. Transforming these terms to the spatial domain results in a first order Sommerfeld integral.

In [4] it was attempted to remove the asymptotic behaviour of the off-diagonal terms by integrating using Sommerfeld identity, i.e. the spectral-spatial pair,

$$
\frac{e^{-\gamma\left|z+z^{\prime}\right|}}{2 \gamma} \leftrightarrow \frac{e^{-j k_{i}\left|\mathbf{r}-\mathbf{r}^{\prime \prime}\right|}}{4 \pi\left|\mathbf{r}-\mathbf{r}^{\prime \prime}\right|}
$$

where $\gamma^{2}=k_{\rho}^{2}-k_{i}^{2}, k_{i}^{2}=k_{0}^{2} \mu_{i} \epsilon_{i}, k_{0}^{2}=\omega^{2} \mu_{0} \epsilon_{0}$, $\mathbf{r}=x \hat{\mathbf{u}}_{x}+y \hat{\mathbf{u}}_{y}+z \hat{\mathbf{u}}_{z}, \mathbf{r}^{\prime \prime}=x^{\prime} \hat{\mathbf{u}}_{x}+y^{\prime} \hat{\mathbf{u}}_{y}-z^{\prime} \hat{\mathbf{u}}_{z}$ for an interface at $z=0$. Note that $\mathbf{r}^{\prime \prime}$ denotes the imaged position of the source. The asymptotic behaviour of this pair, when the source and observer are in the same layer, for $k_{\rho} \rightarrow \infty$ (and $\left.k_{0}=0\right)$ yields the static pair

$$
\frac{e^{-\gamma_{0}\left|z+z^{\prime}\right|}}{2 \gamma_{0}} \leftrightarrow \frac{1}{4 \pi\left|\mathbf{r}-\mathbf{r}^{\prime \prime}\right|}
$$

where $\gamma_{0}=\sqrt{k_{\rho}^{2}}$. Integrating the left-hand side with respect to $z$ results in

$$
\int \frac{e^{-\gamma_{0}\left|z+z^{\prime}\right|}}{2 \gamma_{0}} \mathrm{~d} z=-\operatorname{sgn}\left(z+z^{\prime}\right) \frac{e^{-\gamma_{0}\left|z+z^{\prime}\right|}}{2 \gamma_{0}^{2}},
$$

and the right-hand side with respect to $z$ results in

$$
\begin{aligned}
& \int \frac{1}{4 \pi\left|\mathbf{r}-\mathbf{r}^{\prime \prime}\right|} \mathrm{d} z \\
& =\frac{1}{4 \pi} \operatorname{sgn}\left(z+z^{\prime}\right) \ln \left[\left|z+z^{\prime}\right|+\left|\mathbf{r}-\mathbf{r}^{\prime \prime}\right|\right],
\end{aligned}
$$

which has non-spherical logarithmic singularity.

\section{Spherical singularity at an interface}

Let us define a co-ordinate system such that,

$$
\begin{aligned}
\rho & =\left(x-x^{\prime}\right) \hat{\mathbf{u}}_{x}+\left(y-y^{\prime}\right) \hat{\mathbf{u}}_{y} \\
\mathbf{R} & =\rho \hat{\mathbf{u}}_{\rho}+\left(z+z^{\prime}\right) \hat{\mathbf{u}}_{z} .
\end{aligned}
$$

This allows us to project $\mathbf{R}$ in $\hat{\mathbf{u}}_{\rho}$ (and hence $\hat{\mathbf{u}}_{x}$ and $\hat{\mathbf{u}}_{y}$ ) and $\hat{\mathbf{u}}_{z}$ in terms of angles. The logarithmic singularity can now be written as

$$
\begin{aligned}
& \frac{1}{4 \pi} \operatorname{sgn}\left(z+z^{\prime}\right) \ln \left[\left|z+z^{\prime}\right|+\left|\mathbf{r}-\mathbf{r}^{\prime \prime}\right|\right] \\
& \quad=\frac{1}{4 \pi} \operatorname{sgn}\left(z+z^{\prime}\right) \ln [R \cos \theta+R] \\
& \quad=\frac{1}{4 \pi} \operatorname{sgn}\left(z+z^{\prime}\right)[\ln R+\ln (1+\cos \theta)],
\end{aligned}
$$

where $\theta$ is the angle between $\mathbf{r}-\mathbf{r}^{\prime \prime}$ and the $z$-axis in the range.

In the above equation $\cos \theta$ never becomes a negative value. This is due to the sign term. For example, if the source and the observer are in the top half space, $\theta$ ranges from $-\frac{\pi}{2}$ to $\frac{\pi}{2}$, which produces a non-negative value of $\cos \theta$. For this case, $z$ and $z^{\prime}$ are above the interface (hence positive values) so that the sign term produces a plus sign.

When the source and observer are in the bottom half space, the sign term produces a minus sign as $z$ and $z^{\prime}$ co-ordinates are now below the interface (and hence negative). This minus sign is equivalent to flipping the direction of the $z$-axis. This sign change therefore results in the bottom half space case being a mirror of the top half space case. This means that the range of $\theta$ is again between $-\frac{\pi}{2}$ and $\frac{\pi}{2}$, resulting in a non-negative value for $\cos \theta$.

For the case where source and observer are in adjacent layers, the sign term can be written as $\operatorname{sgn}\left(z-z^{\prime}\right)$ - which can also be mirrored - further preventing $\cos \theta$ from being negative.

The second logarithm becomes singular when $\theta=$ $\pi$. However, this does not apply for our case.

As a result, we are only left with the $\ln R$ term, which is singular. However, this singularity is now spherically symmetric.

\section{Self patch integration}

For the integration of the self patch, where the testing and basis triangles are the same, it is possible 
to rotate the co-ordinate system so the face of the triangle is along a projected plane (an $x, y$-plane). This means that the area $\mathrm{d} A$ is now $\mathrm{d} x \mathrm{~d} y$.

By using the identity

$$
\ln \left(x^{2}+y^{2}\right)=\frac{1}{2} \nabla \cdot\left[\left(x \hat{\mathbf{u}}_{x}+y \hat{\mathbf{u}}_{y}\right) \ln \left(x^{2}+y^{2}\right)\right]-1,
$$

it is possible to write the integral as

$$
\begin{aligned}
& \frac{1}{4} \iint_{T^{+}}\left\{\nabla \cdot\left[\left(x \hat{\mathbf{u}}_{x}+y \hat{\mathbf{u}}_{y}\right) \ln \left(x^{2}+y^{2}\right)\right]-1\right\} \mathrm{d} x \mathrm{~d} y \\
& =\frac{1}{4} \oint_{\partial T_{\text {new }}^{+}}\left[\left(x \hat{\mathbf{u}}_{x}+y \hat{\mathbf{u}}_{y}\right) \ln \left(x^{2}+y^{2}\right)\right] \cdot \underline{\nu} \mathrm{d} \ell \\
& \quad-\frac{1}{4} \operatorname{Area}\left(T_{\text {new }}^{+}\right),
\end{aligned}
$$

where $T_{\text {new }}^{+}$is the new projected area of the triangle, and $\underline{\nu}$ is normal to the $x, y$-plane. As $R$ is the square root of $x^{2}+y^{2}$, there is an extra factor $\frac{1}{2}$ in front of the integral. The area term is a constant, meaning that the line integral is the remaining term that requires evaluation.

For a triangle the integration path of the third edge is given by

$$
\begin{aligned}
& x_{3}(\tau)=x_{1}+\tau\left(x_{2}-x_{1}\right) \\
& y_{3}(\tau)=y_{1}+\tau\left(y_{2}-y_{1}\right)
\end{aligned}
$$

where $\tau$ is the (scaled) integration path along the line, and the subscripts 1 and 2 represent the first and second vertex, respectively. A similar expression can be obtained for the other two edges. This means that for edge 3 ,

$$
\begin{aligned}
x^{2}+y^{2} & =x_{1}^{2}+2 x_{1} \tau\left(x_{2}-x_{1}\right)+\tau^{2}\left(x_{2}-x_{1}\right)^{2} \\
& +y_{1}^{2}+2 y_{1} \tau\left(y_{2}-y_{1}\right)+\tau^{2}\left(y_{2}-y_{1}\right)^{2}
\end{aligned}
$$

meaning that the integration for this edge is of the form

$$
\begin{aligned}
& \frac{1}{4} \int_{\ell_{3}}\left[\left(x \hat{\mathbf{u}}_{x}+y \hat{\mathbf{u}}_{y}\right) \ln \left(x^{2}+y^{2}\right)\right] \cdot \underline{\nu} \mathrm{d} \ell \\
& =\frac{1}{4} \int_{\tau=0}^{1} \ln \left[a_{3} \tau^{2}+b_{3} \tau+c_{3}\right] \\
& \quad \times\left\{\left[x_{1}+\tau\left(x_{2}-x_{1}\right)\right] \nu_{x}\right. \\
& \left.\quad+\left[y_{1}+\tau\left(y_{2}-y_{1}\right)\right] \nu_{y}\right\} L \mathrm{~d} \tau,
\end{aligned}
$$

where $L$ is the line segment length for edge 3. Furthermore, $\nu_{x}$ and $\nu_{x}$ are constant for the edge. The variables $a_{3}, b_{3}$ and $c_{3}$ are real. A similar expression can be obtained for the other two edges by changing the vertex indices in the above expression. This definite integral therefore has the solution

$$
\begin{aligned}
& \int_{\tau=0}^{1} \ln \left[a \tau^{2}+b \tau+c\right](d+e \tau) \mathrm{d} \tau \\
& =\frac{1}{4 a^{2}} \ln \left[\frac{(a+b+c)^{\left[2 a^{2}(2 d+e)+2 a(b d+e c)-e b^{2}\right]}}{c^{\left[2 a(b d+e c)-e b^{2}\right]}}\right] \\
& -\frac{1}{2 a}(4 a d+e a-c b) \\
& -\frac{\left(b^{2}-4 a c\right)^{1 / 2}(e b-2 a d)}{2 a^{2}} \tan ^{-1}\left[\frac{(4 a c-b)^{1 / 2}}{2 c-b}\right],
\end{aligned}
$$

where $d$ and $e$ are also real.

\section{Conclusion}

An alternative approach that results in a spherically symmetric singularity for the off-diagonal terms of the dyadic Green function is derived. Using this approach produces a uniform (orientation independent) singular term, which is more flexible to implement numerically. Finally, a discussion on how the self patch integration results in a definite integral is given.

\section{Acknowledgments}

This research is supported by the Dutch Technology Foundation STW, which is part of the Netherlands Organisation for Scientific Research (NWO) and is partly funded by the Ministry of Economic Affairs.

\section{References}

[1] S.M. Rao, D. Wilton, and A.W. Glisson. Electromagnetic scattering by surfaces of arbitrary shape. Antennas and Propagation, IEEE Transactions on, 30(3):409-418, May 1982.

[2] K.A. Michalski and D. Zheng. Electromagnetic scattering and radiation by surfaces of arbitrary shape in layered media. i. theory. Antennas and Propagation, IEEE Transactions on, 38(3):335344, Mar 1990.

[3] K.A. Michalski and J.R. Mosig. Multilayered media green's functions in integral equation formulations. Antennas and Propagation, IEEE Transactions on, 45(3):508-519, Mar 1997.

[4] K.A. Michalski and D. Zheng. Electromagnetic scattering and radiation by surfaces of arbitrary shape in layered media. ii. implementation and results for contiguous half-spaces. Antennas and Propagation, IEEE Transactions on, 38(3):345352, Mar 1990. 
[5] M. Vrancken and G. A. E. Vandenbosch. Semantics of dyadic and mixed potential field representation for 3 -d current distributions in planar stratified media. IEEE Transactions on Antennas and Propagation, 51(10):2778-2787, Oct 2003. 